\title{
Tenofovir Alafenamide
}

\author{
National Cancer Institute
}

\section{Source}

National Cancer Institute. Tenofovir Alafenamide. NCI Thesaurus. Code C148276.

A lipophilic phosphonamidate prodrug of tenofovir, a synthetic antiviral acyclic nucleotide analog of adenosine 5-monophosphate and a nucleoside reverse transcriptase inhibitor (NRTI), with antiviral activity against hepatitis B virus (HBV) and potentially against human immunodeficiency virus (HIV). Upon oral administration, tenofovir alafenamide is taken up by hepatocytes through passive diffusion and through the hepatic uptake transporters organic anion transporting polypeptides 1B1 (OATP1B1) and 1B3 (OATP1B3). Inside the hepatocytes, tenofovir alafenamide is hydrolyzed and converted to tenofovir by carboxylesterase 1 (CES1). Intracellular tenofovir is phosphorylated by cellular kinases to its pharmacologically active form, tenofovir diphosphate. Tenofovir diphosphate is incorporated into viral DNA instead of the natural substrate deoxyadenosine 5-triphosphate, and inhibits HBV reverse transcriptase, resulting in DNA chain-termination and inhibition of HBV replication. In addition, tenofovir diphosphate is incorporated into HIV DNA instead of the natural substrate deoxyadenosine 5triphosphate, thereby inhibiting HIV-1 reverse transcriptase (RT) and resulting in DNA chain termination and impairment of HIV replication. 